\title{
Some coupled fixed point theorems in partially ordered S-metric spaces
}

\author{
Vishal Gupta and Raman Deep
}




\title{
SOME COUPLED FIXED POINT THEOREMS IN PARTIALLY ORDERED S-METRIC SPACES
}

\author{
VISHAL GUPTA AND RAMAN DEEP
}

Received 18 February, 2014

\begin{abstract}
In this paper, we prove some fixed point results using mixed weakly monotone property and altering distance function in the setting of S-metric space. Our result generalizes the result of Lakshmikantham and Ciric [V. Lakshmikantham and L. Ciric, Coupled common fixed point theorems for nonlinear contractions in partially ordered metric spaces, Nonlinear Analysis, 70, (2009), 4341 - 4349].
\end{abstract}

2010 Mathematics Subject Classification: 54H25; 47H10

Keywords: fixed point, S-metric space, partially ordered space, mixed weakly monotone property

\section{INTRODUCTION}

In functional analysis, the concept of space is very broad and astonishing. A space will be a set of unspecified elements satisfying certain axioms, and by choosing different set of axioms, we shall obtain different type of spaces. In partially ordered set, as the name indicates, ordering and sequencing is defined between the elements of a set. Although there are some elements in the set which are not related. A set in which all elements are related is called totally ordered set. In [18] Marr defines the concept of convergence in partially ordered metric space. Also, he tried to obtain a relation between metric space and partially ordered metric space, and claimed that the fixed point theorems in metric spaces are the particular cases of fixed point results in partially ordered metric spaces.

Definition 1 ([18]). A partially ordered space is a set $X$ with a binary relation $\leq$, which satisfy the three conditions:

(1) $x \leq x$ for all $x \in X$;

(2) $x \leq y$ and $y \leq z$ implies $x \leq z$ for all $x, y, z \in X$;

(3) $x \leq y$ and $y \leq x$ implies $x=y$ for all $x, y \in X$.

There is vast literature of obtaining fixed points using different contractive conditions. In this direction, Dolbosco [3] took initiation to gave fixed point results using altering distance function or control function. Altering distance function measures 
the distance between two points. Khan et. al. [15] worked on the concept of altering distance between points, for pair of self mappings. Also, Altun [1], Aydi [2], Gupta et al [8,9], Iseki [11] established theorems on sequence of self mappings with altering distance function.

Definition 2 ([15]). An altering distance function is a function $\psi:[0, \infty) \rightarrow$ $[0, \infty)$, which is

(1) monotone increasing and continuous,

(2) $\psi(t)=0$ iff $t=0$.

In 1963, Gahler [6] introduced the generalization of metric space and called it 2metric space. Fixed point theorems in 2-metric space has been proved initially by Iseki [12]. After that several authors proved fixed point results in the setting of 2metric space. In 1979, Fisher [5] gave common fixed point results using commuting mapping. Jungck [13] and Kubiak [16] also proved some results using commuting and semi-commuting mappings. In 1992, Murthy [19] used compatible type mapping to find fixed point, which is more general than commuting and semi-commuting maps. After that in 1978, Khan [14] proved a result by taking a uniformly convergent sequence of 2-metrics in X. In 1988, Ha et al [10] objected on Gahler theory that 2-metric space is not the generalization of metric space. He claimed that the contraction mappings in these spaces are unrelated. These considerations inspired Dhage [4] to introduce a new metric namely D-metric. After that Sedghi and Shobe [22] gave $D^{*}$ metric space which is more general than D-metric space. Further the topological properties of D-metric space are objected by Mustafa [20] in 2003, and Mustafa [21] gave a more generalized metric called G-metric. He defined many concepts like convergence, continuity, completeness, compactness, product of spaces in the setting of G-metric space and stated that every G-metric space is topologically equivalent to a metric space.

Definition 3 ([21]). Let $\mathrm{X}$ be a non-empty set and $G: X \times X \times X \rightarrow[0, \infty)$ be a function satisfying the following conditions for all $x, y, z, a \in X$,

(1) $G(x, y, z)=0$ if $x=y=z$;

(2) $0<G(x, x, y)$ for all $x, y \in X$ with $x \neq y$;

(3) $G(x, x, y) \leq G(x, y, z)$ for all $x, y, z \in X$ with $x \neq y$;

(4) $G(x, y, z)=G(x, z, y)=G(y, z, x) \ldots$;

(5) $G(x, y, z) \leq G(x, a, a)+G(a, y, z)$ for all $x, y, z, a \in X$.

Then the function $\mathrm{G}$ is called $\mathrm{G}$-metric on $\mathrm{X}$ and the pair $(X, G)$ is called a $\mathrm{G}$-metric space.

In 2011, Sedghi and Shobe [23] introduced a more general space namely S-metric space which is generalization of $D^{*}$ metric space and G-metric space. 
Definition 4 ([23]). Let $X$ be a non-empty set. A generalized metric or S-metric on $\mathrm{X}$ is a function, $S: X \times X \times X \rightarrow[0, \infty)$ that satisfies the following conditions for all $x, y, z, a \in X$,

(1) $S(x, y, z) \geq 0$

(2) $S(x, y, z)=0$ iff $x=y=z$;

(3) $S(x, y, z) \leq S(a, y, z)+S(a, x, x)$.

Then the pair $(X, S)$ is called a $S$-metric space.

Example 1 ([23]). If $X=R^{n}$, define

(1) $S(x, y, z)=\|y+x-2 z\|+\|y-z\|$

(2) $S(x, y, z)=d(x, y)+d(x, z)$, here $d$ is the ordinary metric on X.

Then $(X, S)$ is $\mathrm{S}$-metric space.

Definition 5 ([23]). Let $(X, S)$ be a S-metric space and $A \subset X$,

(1) If for every $x \in A$, there exists $r>0$ such that $B_{S}(x, r) \subset A$, then subset $\mathrm{A}$ is called open subset of $\mathrm{X}$.

(2) Subset $\mathrm{A}$ of $\mathrm{X}$ is said to be S-bounded if there exists $r>0$ such that $S(x, y, y)<r$ for all $x, y \in A$.

(3) A sequence $\left\{x_{n}\right\}$ in $\mathrm{X}$ converges to $x$ if and only if $S\left(x_{n}, x, x\right)$ $=S\left(x, x_{n}, x_{n}\right) \rightarrow 0$ as $n \rightarrow \infty$. That is, for each $\epsilon>0$, there exists $n_{0} \in N$ such that $S\left(x, x_{n}, x_{n}\right)<\epsilon$ for all $n \geq n_{0}$.

(4) A sequence $\left\{x_{n}\right\}$ in $X$ is called a Cauchy sequence if for each $\epsilon>0$, there exists $n_{0} \in N$ such that $S\left(x_{n}, x_{m}, x_{m}\right)<\epsilon$ for each $n, m \geq n_{0}$.

The $\mathrm{S}$-metric space $(X, S)$ is said to be complete if every Cauchy sequence is convergent.

(5) Let $\tau$ be the set of all $A \subset X$ with $x \in A$ if and only if there exists $r>0$ such that $B_{S}(x, r) \subset A$. Then $\tau$ is a topology induced by the S-metric $\mathrm{S}$ on X.

Also, Sedghi et. al. [24] gave some properties of S-metric space and proved fixed point theorems in this space. Nguyen [25] proved coupled fixed point in partially ordered S-metric space with the help of mixed weakly monotone maps.

Lakshmikantham and Ciric [17] introduced the concept of mixed g-monotone mapping and proved coincidence fixed point theorems in partially ordered metric space.

Definition 6 ([17]). Let $(X, \leq)$ be a partially ordered set and $F: X \times X \rightarrow X$ and $g: X \rightarrow X$. We say $\mathrm{F}$ has mixed g-monotone property if $\mathrm{F}$ is monotone $\mathrm{g}$ non-decreasing in its first argument, and is monotone g-non-increasing in its second argument, that is, for any $x, y \in X$,

$$
\begin{aligned}
x_{1}, x_{2} & \in X, g\left(x_{1}\right) \leq g\left(x_{2}\right) \text { implies } F\left(x_{1}, y\right) \leq F\left(x_{2}, y\right) \\
\text { and } y_{1}, y_{2} \in X, g\left(y_{1}\right) & \leq g\left(y_{2}\right) \text { implies } F\left(x, y_{1}\right) \geq F\left(x, y_{2}\right) .
\end{aligned}
$$


Theorem 1 ([17]). Let $(X, \leq)$ be a partially ordered set and $F: X \times X \rightarrow X$ and suppose there is a metric $d$ on $X$ such that $(X, d)$ is a complete metric space. Assume that there is a function $\psi:[0, \infty) \rightarrow[0, \infty)$ with $\psi(t)<t$ and

$$
\lim _{r \rightarrow t^{+}} \phi(r)<t \text { for each } t>0 .
$$

Also suppose $F: X \times X \rightarrow X$ and $g: X \rightarrow X$ are such that $F$ has the mixed $g$ monotone property satisfying:

$$
d(F(x, y), F(u, v)) \leq \phi\left(\frac{d(g(x), g(u))+d(g(y), g(v))}{2}\right),
$$

for all $x, y, u, v \in X$, for which $g(x) \leq g(u)$ and $g(y) \geq g(v)$. Consider $F(X \times X) \subseteq$ $g(X), g$ is continuous and commutes with $F$ and also suppose either

(1) $F$ is continuous

or

(2) X has the following property:

(a) if a non-decreasing sequence $\left\{x_{n}\right\} \rightarrow x$, then $x_{n} \leq x$ for all $n$;

(b) if a non-increasing sequence $\left\{y_{n}\right\} \rightarrow y$, then $y \leq y_{n}$ for all $n$.

If there exist $x_{0}, y_{0} \in X$ such that $g\left(x_{0}\right) \leq F\left(x_{0}, y_{0}\right)$ and $g\left(y_{0}\right) \geq F\left(y_{0}, x_{0}\right)$

then there exist $x, y \in X$ such that $g(x)=F(x, y)$ and $g(y)=F(y, x)$.

In 2012, Gordji et. al. [7] introduced the concept of the mixed weakly inceasing property of mappings and proved a coupled fixed point result.

Definition 7 ([7]). Let $(X, \leq)$ be a partially ordered set and $f, g: X \times X \rightarrow X$ be mappings. We say that a pair $(f, g)$ has the mixed weakly monotone property on $\mathrm{X}$, if for any $x, y \in X$

$$
x \leq f(x, y), y \geq f(y, x),
$$

this implies

$$
f(x, y) \leq g(f(x, y), f(y, x)), f(y, x) \geq g(f(y, x), f(x, y))
$$

and

$$
x \leq g(x, y), y \geq g(y, x),
$$

this implies

$$
\Rightarrow g(x, y) \leq f(g(x, y), g(y, x)), g(y, x) \geq f(g(y, x), g(x, y)) .
$$

Theorem 2 ([7]). Let $(X, \leq, d)$ be a partially ordered complete metric space. Let $f, g: X \times X \rightarrow X$ are mappings such that pair $(f, g)$ has the mixed weakly monotone property on $X$. Suppose that there exist $p, q, r, s \geq 0$ with $p+q+r+2 s<1$ such that 


$$
\begin{aligned}
d(f(x, y), g(u, v)) \leq & \frac{p}{2} D((x, y),(u, v)) \\
& +\frac{q}{2} D((x, y),(f(x, y), f(y, x))) \\
& +\frac{r}{2} D((u, v),(g(u, v), g(v, u))) \\
& +\frac{s}{2} D((x, y),(g(u, v), g(v, u))) \\
& +\frac{s}{2} D((u, v),(f(x, y), f(y, x))),
\end{aligned}
$$

for all $x, y, u, v \in X$ with $x \leq u$ and $y \geq v$. Let $x_{0}, y_{0} \in X$ be such that $x_{0} \leq$ $f\left(x_{0}, y_{0}\right), y_{0} \geq f\left(y_{0}, x_{0}\right)$ or $x_{0} \leq g\left(x_{0}, y_{0}\right), y_{0} \geq g\left(y_{0}, x_{0}\right)$.

If $f$ or $g$ is continuous, then $f$ and $g$ have a coupled common fixed point in $X$.

This result is generalized by Nguyen [25] to S-metric space as follows:

Theorem 3 ([25]). Let $(X, \leq, S)$ be a partially ordered S-metric space and $f, g$ : $X \times X \rightarrow X$ be two maps such that

(1) $X$ is complete;

(2) The pair $(f, g)$ has the mixed weakly monotone property on $X, x_{0} \leq f\left(x_{0}, y_{0}\right)$, $f\left(y_{0}, x_{0}\right) \leq y_{0}$ or $x_{0} \leq g\left(x_{0}, y_{0}\right), g\left(y_{0}, x_{0}\right) \leq y_{0}$ for some $x_{0}, y_{0} \in X$;

(3) There exist $p, q, r, s \geq 0$ satisfying $p+q+r+2 s<1$ and

$$
\begin{aligned}
d(f(x, y), f(x, y), g(u, v)) \leq & \frac{p}{2} D((x, y),(x, y),(u, v)) \\
& +\frac{q}{2} D((x, y),(x, y),(f(x, y), f(y, x))) \\
& +\frac{r}{2} D((u, v),(u, v),(g(u, v), g(v, u))) \\
& +\frac{s}{2} D((x, y),(x, y),(g(u, v), g(v, u))) \\
& +\frac{s}{2} D((u, v),(u, v),(f(x, y), f(y, x))),
\end{aligned}
$$

for all $x, y, u, v \in X$ with $x \leq u$ and $y \geq v$, where $D=((x, y),(u, v),(z, w))=S(x, u, z)+S(y, v, w)$;

(4) for $g$ is continuous or $X$ has the following property:

(a) If $\left\{x_{n}\right\}$ is an increasing sequence with $x_{n} \rightarrow x$, then $x_{n} \leq x$ for all $n \in N$,

(b) If $\left\{x_{n}\right\}$ is an decreasing sequence with $x_{n} \rightarrow x$, then $x \leq x_{n}$ for all $n \in N$.

Then $f$ and $g$ have a coupled common fixed point in X. 
Now our aim is to prove new common coupled fixed points theorems by using altering distance function and mixed weakly monotone maps in partially ordered Smetric space.

\section{MAIN RESULT}

Theorem 4. Let $(X, \leq, S)$ be a partially ordered complete $S$-metric space and the mappings $f, g: X \times X \rightarrow X$ satisfies the mixed weakly monotone property on $X ; x_{0} \leq$ $f\left(x_{0}, y_{0}\right), f\left(y_{0}, x_{0}\right) \leq y_{0}$ or $x_{0} \leq g\left(x_{0}, y_{0}\right), g\left(y_{0}, x_{0}\right) \leq y_{0}$ for some $x_{0}, y_{0} \in X$. Consider a function $\phi:[0, \infty) \rightarrow[0, \infty)$ with $\phi(t)<t$ and

such that

$$
\lim _{r \rightarrow t^{+}} \phi(r)<t \text { for each } t>0 .
$$

$$
S(f(x, y), f(x, y), g(u, v)) \leq \phi\left(\frac{S(x, x, u)+S(y, y, v)}{2}\right) ;
$$

$\forall x, y, u, v \in X$ with $x \leq u$ and $y \geq v$.

Also, assume that either $f$ or $g$ is continuous or $X$ has the following property:

(1) If $\left\{x_{n}\right\}$ is an increasing sequence with $x_{n} \rightarrow x$ then $x_{n} \leq x$ for all $n \in N$;

(2) If $\left\{y_{n}\right\}$ is an decreasing sequence with $y_{n} \rightarrow y$ then $y \leq y_{n}$ for all $n \in N$.

Then $f$ and $g$ have a coupled common fixed point in $X$.

Proof. Consider $x_{0} \leq f\left(x_{0}, y_{0}\right)$ and $y_{0} \geq f\left(y_{0}, x_{0}\right)$ and let $f\left(x_{0}, y_{0}\right)=x_{1}$ and $f\left(y_{0}, x_{0}\right)=y_{1}$.

Now, $x_{1}=f\left(x_{0}, y_{0}\right) \leq g\left(f\left(x_{0}, y_{0}\right), f\left(y_{0}, x_{0}\right)\right)=g\left(x_{1}, y_{1}\right)=x_{2}$ (say) and $y_{1}=$ $f\left(y_{0}, x_{0}\right) \leq g\left(f\left(y_{0}, x_{0}\right), f\left(x_{0}, y_{0}\right)\right)=g\left(y_{1}, x_{1}\right)=y_{2}$ (say).

Continuing in this way, we have $x_{2 n+1}=f\left(x_{2 n}, y_{2 n}\right), y_{2 n+1}=f\left(y_{2 n}, x_{2 n}\right)$ and $x_{2 n+2}=g\left(x_{2 n+1}, y_{2 n+1}\right), y_{2 n+2}=g\left(y_{2 n+1}, x_{2 n+1}\right)$.

Thus, we conclude that $\left\{x_{n}\right\}$ is increasing and $\left\{y_{n}\right\}$ is decreasing sequence.

Similarly, from the condition $x_{0} \leq g\left(x_{0}, y_{0}\right)$ and $y_{0} \geq g\left(y_{0}, x_{0}\right)$, we can say that the sequences $\left\{x_{n}\right\}$ and $\left\{y_{n}\right\}$ are increasing or decreasing.

Now from (2.1), we obtain

$$
\begin{aligned}
S\left(f\left(x_{2 n}, y_{2 n}\right), f\left(x_{2 n}, y_{2 n}\right), g\left(x_{2 n+1}, y_{2 n+1}\right)\right) \\
\leq \phi\left(\frac{S\left(x_{2 n}, x_{2 n}, x_{2 n+1}\right)+S\left(y_{2 n}, y_{2 n}, y_{2 n+1}\right)}{2}\right),
\end{aligned}
$$

which implies,

$$
\begin{aligned}
& S\left(x_{2 n+1}, x_{2 n+1}, x_{2 n+2}\right) \\
& \leq \phi\left(\frac{S\left(x_{2 n}, x_{2 n}, x_{2 n+1}\right)+S\left(y_{2 n}, y_{2 n}, y_{2 n+1}\right)}{2}\right) .
\end{aligned}
$$

Again, using the same step, we have 


$$
\begin{aligned}
& S\left(y_{2 n+1}, y_{2 n+1}, y_{2 n+2}\right) \\
& \leq \phi\left(\frac{S\left(y_{2 n}, y_{2 n}, y_{2 n+1}\right)+S\left(x_{2 n}, x_{2 n}, x_{2 n+1}\right)}{2}\right) .
\end{aligned}
$$

Adding (2.2) and (2.3), we get

$$
\begin{aligned}
S\left(x_{2 n+1}, x_{2 n+1}, x_{2 n+2}\right)+ & S\left(y_{2 n+1}, y_{2 n+1}, y_{2 n+2}\right) \\
& \leq 2 \phi\left(\frac{S\left(x_{2 n}, x_{2 n}, x_{2 n+1}\right)+S\left(y_{2 n}, y_{2 n}, y_{2 n+1}\right)}{2}\right) .
\end{aligned}
$$

Thus,

$$
t_{2 n} \leq 2 \phi\left(\frac{t_{2 n-1}}{2}\right),
$$

where $t_{2 n}=S\left(x_{2 n+1}, x_{2 n+1}, x_{2 n+2}\right)+S\left(y_{2 n+1}, y_{2 n+1}, y_{2 n+2}\right)$.

Now, using the property that $\phi(t)<t, t>0$ in (2.4), this gives

$$
t_{2 n} \leq 2\left(\frac{t_{2 n-1}}{2}\right)=t_{2 n-1} \text {. }
$$

Interchanging the role of mappings $f$ and $g$, and using (2.1), we have

$$
\begin{aligned}
& S\left(g\left(x_{2 n+1}, y_{2 n+1}\right), g\left(x_{2 n+1}, y_{2 n+1}\right), f\left(x_{2 n+2}, y_{2 n+2}\right)\right) \\
& \leq \phi\left(\frac{S\left(x_{2 n+1}, x_{2 n+1}, x_{2 n+2}\right)+S\left(y_{2 n+1}, y_{2 n+1}, y_{2 n+2}\right)}{2}\right) .
\end{aligned}
$$

Proceeding as above one can get, $t_{2 n+1} \leq 2 \phi\left(\frac{t_{2 n}}{2}\right)$.

Again using the fact that $\phi(t)<t$ above inequality gives

$$
t_{2 n+1} \leq t_{2 n}
$$

From (2.5) and (2.6), we conclude that $\left\{t_{n}\right\}$ is a decreasing sequence. Therefore there exists some $t \geq 0$ such that

$$
\lim _{n \rightarrow \infty} t_{n}=t
$$

Also, using the properties of function $\phi$

$$
t=\lim _{n \rightarrow \infty} t_{2 n} \leq 2 \lim _{n \rightarrow \infty} \phi\left(\frac{t_{2 n-1}}{2}\right)<t,
$$

This is a contradiction. Hence $t=0$.

That is,

$$
\lim _{n \rightarrow \infty} S\left(x_{n}, x_{n}, x_{n+1}\right)+S\left(y_{n}, y_{n}, y_{n+1}\right)=0 .
$$

Using property of S-metric space, for $n \leq m$, we have

$$
\begin{aligned}
S\left(x_{n}, x_{n}, x_{m}\right)+S\left(y_{n}, y_{n}, y_{m}\right) \leq & 2 S\left(x_{n}, x_{n}, x_{n+1}\right)+S\left(x_{n+1}, x_{n+1}, x_{m}\right) \\
& +2 S\left(y_{n}, y_{n}, y_{n+1}\right)+S\left(y_{n+1}, y_{n+1}, y_{m}\right) .
\end{aligned}
$$


on taking limit as $n, m \rightarrow \infty$ and using (2.7), we obtain

$$
\begin{aligned}
\lim _{n, m \rightarrow \infty}\left[S\left(x_{n}, x_{n}, x_{m}\right)+S\left(y_{n}, y_{n}, y_{m}\right)\right] \\
\leq \lim _{n, m \rightarrow \infty}\left[S\left(x_{n+1}, x_{n+1}, x_{m}\right)+S\left(y_{n+1}, y_{n+1}, y_{m}\right)\right] \\
\leq \lim _{n, m \rightarrow \infty}\left[2 S\left(x_{n+1}, x_{n+1}, x_{n+2}\right)+S\left(x_{n+2}, x_{n+2}, x_{m}\right)\right. \\
\left.\quad+2 S\left(y_{n+1}, y_{n+1}, y_{n+2}\right)+S\left(y_{n+2}, y_{n+2}, y_{m}\right)\right] \\
=\lim _{n, m \rightarrow \infty}\left[S\left(x_{n+2}, x_{n+2}, x_{m}\right)+S\left(y_{n+2}, y_{n+2}, y_{m}\right)\right] .
\end{aligned}
$$

By repeatedly use of property of S-metric space, we get

$$
\begin{aligned}
\lim _{n, m \rightarrow \infty}\left[S\left(x_{n}, x_{n}, x_{m}\right)+S\left(y_{n}, y_{n}, y_{m}\right)\right] \\
\leq \lim _{n, m \rightarrow \infty}\left[2 S\left(x_{m-1}, x_{m-1}, x_{m}\right)+S\left(x_{m}, x_{m}, x_{m+1}\right)\right. \\
\left.+2 S\left(y_{m-1}, y_{m-1}, y_{m}\right)+S\left(y_{m}, y_{m}, y_{m+1}\right)\right],
\end{aligned}
$$

which gives,

$$
\lim _{n, m \rightarrow \infty}\left[S\left(x_{n}, x_{n}, x_{m}\right)+S\left(y_{n}, y_{n}, y_{m}\right)\right]=0 .
$$

This shows that $\left\{x_{n}\right\}$ and $\left\{y_{n}\right\}$ are two Cauchy sequences in $X$. Since $X$ is complete, $\exists x, y \in X$ such that

$$
x_{n} \rightarrow x \text { and } y_{n} \rightarrow y \text { as } n \rightarrow \infty .
$$

As $f$ is supposed to be continuous, therefore

$$
\begin{aligned}
x & =\lim _{n \rightarrow \infty} x_{2 n+1}=\lim _{n \rightarrow \infty} f\left(x_{2 n}, y_{2 n}\right) \\
& =f\left(\lim _{n \rightarrow \infty} x_{2 n}, \lim _{n \rightarrow \infty} y_{2 n}\right)=f(x, y)
\end{aligned}
$$

and

$$
\begin{aligned}
y & =\lim _{n \rightarrow \infty} y_{2 n+1}=\lim _{n \rightarrow \infty} f\left(y_{2 n}, x_{2 n}\right) \\
& =f\left(\lim _{n \rightarrow \infty} y_{2 n}, \lim _{n \rightarrow \infty} x_{2 n}\right)=f(y, x) .
\end{aligned}
$$

From (2.1),

$$
\begin{aligned}
& S(f(x, y), f(x, y), g(x, y))+S(f(y, x), f(y, x), g(y, x)) \\
& \leq \phi\left(\frac{S(x, x, x)+S(y, y, y)}{2}\right)+\phi\left(\frac{S(y, y, y)+S(x, x, x)}{2}\right), \\
& S(x, x, g(x, y))+S(y, y, g(y, x))=0 .
\end{aligned}
$$


Thus we have, $g(x, y)=x$ and $g(y, x)=y$.

Hence $(x, y)$ is coupled common fixed point of $\mathrm{f}$ and $\mathrm{g}$.

Similarly, the result follows, when $\mathrm{g}$ is assumed to be continuous.

Now, consider the other assumption that for an increasing sequence $\left\{x_{n}\right\}$ with $x_{n} \rightarrow x$ we have $x_{n} \leq x$ and for decreasing sequence $\left\{y_{n}\right\}$ with $y_{n} \rightarrow y$ we have $y \leq y_{n}$ for all $n \in N$.

Consider,

$$
\begin{aligned}
S(x, x, f(x, y)) & \leq 2 S\left(x, x, x_{n}\right)+S\left(f(x, y), f(x, y), x_{n}\right) \\
& =2 S\left(x, x, x_{n}\right)+S\left(f(x, y), f(x, y), g\left(x_{n-1}, y_{n-1}\right)\right) \\
& \leq 2 S\left(x, x, x_{n}\right)+\phi\left(\frac{S\left(x, x, x_{n-1}\right)+S\left(y, y, y_{n-1}\right)}{2}\right) .
\end{aligned}
$$

Similarly,

$$
\begin{aligned}
S(y, y, f(y, x)) & \leq 2 S\left(y, y, y_{n}\right)+S\left(f(y, x), f(y, x), y_{n}\right) \\
& =2 S\left(y, y, y_{n}\right)+S\left(f(y, x), f(y, x), g\left(y_{n-1}, x_{n-1}\right)\right) \\
& \leq 2 S\left(y, y, y_{n}\right)+\phi\left(\frac{S\left(y, y, y_{n-1}\right)+S\left(x, x, x_{n-1}\right)}{2}\right) .
\end{aligned}
$$

Adding the above inequalities, one can get

$$
\begin{aligned}
S(x, x, & f(x, y))+S(y, y, f(y, x)) \\
& \leq 2 S\left(x, x, x_{n}\right)+2 S\left(y, y, y_{n}\right)+2 \phi\left(\frac{S\left(x, x, x_{n-1}\right)+S\left(y, y, y_{n-1}\right)}{2}\right),
\end{aligned}
$$

on taking $n \rightarrow \infty$, we get $S(x, x, f(x, y))+S(y, y, f(y, x))<0$, which shows that $f(x, y)=x$ and $f(y, x)=y$. By interchanging the role of functions $\mathrm{f}$ and $\mathrm{g}$, we get the same result for $\mathrm{g}$. Thus $(x, y)$ is the common coupled fixed point of $\mathrm{f}$ and $\mathrm{g}$.

Corollary 1. Let $(X, \leq, S)$ be a partially ordered complete $S$-metric space and the mappings $f, g: X \times X \rightarrow X$ satisfies the mixed weakly monotone property on $X$. Consider a function $\phi:[0, \infty) \rightarrow[0, \infty)$ with $\phi(t)<t$ and

$$
\lim _{r \rightarrow t^{+}} \phi(r)<t \text { for each } t>0
$$

such that

$$
S(f(x, y), f(x, y), g(u, v)) \leq \frac{a}{2}[S(x, x, u)+S(y, y, v)],
$$

$\forall x, y, u, v \in X$ with $x \leq u$ and $y \geq v$.

Also, assume that either for $g$ is continuous or $X$ has the following property:

(1) If $\left\{x_{n}\right\}$ is an increasing sequence with $x_{n} \rightarrow x$ then $x_{n} \leq x$ for all $n \in N$;

(2) If $\left\{y_{n}\right\}$ is an decreasing sequence with $y_{n} \rightarrow y$ then $y \leq y_{n}$ for all $n \in N$.

Proof. Taking $\phi(t)=a t$ with $a \in(0,1)$ in Theorem 4 , we obtain the result. 
Corollary 2. Let $(X, \leq, S)$ be a partially ordered complete $S$-metric space and the mapping $f: X \times X \rightarrow X$ satisfies the mixed monotone property on $X$. Consider a function $\phi:[0, \infty) \rightarrow[0, \infty)$ with $\phi(t)<t$

$$
\lim _{r \rightarrow t^{+}} \phi(r)<t \text { for each } t>0
$$

such that

$$
S(f(x, y), f(x, y), f(u, v)) \leq \frac{a}{2}[S(x, x, u)+S(y, y, v)],
$$

$\forall x, y, u, v \in X$ with $x \leq u$ and $y \geq v$.

Also assume that either $f$ is continuous or $X$ has the following property:-

(1) If $\left\{x_{n}\right\}$ is an increasing sequence with $x_{n} \rightarrow x$ then $x_{n} \leq x$ for all $n \in N$;

(2) If $\left\{y_{n}\right\}$ is an decreasing sequence with $y_{n} \rightarrow y$ then $y \leq y_{n}$ for all $n \in N$.

Proof. Taking $\phi(t)=a t$ with $a \in(0,1)$ and $f=g$ in Theorem 4, we obtain the corollary or result.

Corollary 3. Assume that $X$ is totally ordered set in addition to the hypothesis of Theorem 4. Then $f$ and $g$ have unique common fixed point.

Proof. From Theorem 4, $\mathrm{f}$ and g have a coupled common fixed point $(x, y)$. Let $(l, m)$ be another coupled common fixed point of $\mathrm{f}$ and $\mathrm{g}$. Without loss of generality we may assume that $(x, y) \leq(l, m)$. Then from $(2.1)$ we have,

$$
\begin{array}{r}
S(f(x, y), f(x, y), g(l, m)) \leq \phi\left(\frac{S(x, x, l)+S(y, y, m)}{2}\right), \\
S(x, x, l) \leq \phi\left(\frac{S(x, x, l)+S(y, y, m)}{2}\right) .
\end{array}
$$

Similarly,

$$
S(y, y, m) \leq \phi\left(\frac{S(y, y, m)+S(x, x, l)}{2}\right) .
$$

On adding the above inequalities, we have

$$
S(x, x, l)+S(y, y, m) \leq 2 \phi\left(\frac{S(x, x, l)+S(y, y, m)}{2}\right) .
$$

Using the definition of function $\phi$, we get

$$
S(x, x, l)+S(y, y, m)<S(x, x, l)+S(y, y, m),
$$

which is a contradiction. Hence $x=l$ and $y=m$. This proves that the coupled common fixed point of $\mathrm{f}$ and $\mathrm{g}$ is unique.

Again from (2.1), we have

$$
S(f(x, y), f(x, y), g(y, x)) \leq \phi\left(\frac{S(x, x, y)+S(y, y, x)}{2}\right),
$$




$$
S(x, x, y) \leq \phi\left(\frac{S(x, x, y)+S(y, y, x)}{2}\right) .
$$

Similarly,

$$
S(y, y, x) \leq \phi\left(\frac{S(y, y, x)+S(x, x, y)}{2}\right) .
$$

On adding the above inequalities, we have

$$
S(x, x, y)+S(y, y, x) \leq 2 \phi\left(\frac{S(x, x, l)+S(y, y, m)}{2}\right) .
$$

Thus we get $x=y$.

Theorem 5. Let $(X, \leq, S)$ be a partially ordered complete $S$-metric space and the mappings $f, g: X \times X \rightarrow X$ satisfies the mixed weakly monotone property on $X$. Consider a function $\phi:[0, \infty) \rightarrow[0, \infty)$ with $\phi(t)<t$ and

$$
\lim _{r \rightarrow t^{+}} \phi(r)<t \text { for each } t>0
$$

such that

$$
\int_{0}^{S(f(x, y), f(x, y), g(u, v))} \varphi(t) d t \leq \phi \int_{0}^{\frac{S(x, x, u)+S(y, y, v)}{2}} \varphi(t) d t,
$$

$\forall x, y, u, v \in X$ with $x \leq u$ and $y \geq v$. Here $\varphi:[0, \infty) \rightarrow[0, \infty)$ is a Lebesgue integrable function as a summable for each compact $R^{+}$, non-negative and such that for each $\epsilon>0, \int \varphi(t) d t>0$.

Also, assume that either $f$ or $g$ is continuous or $X$ has the following property:

(1) If $\left\{x_{n}\right\}$ is an increasing sequence with $x_{n} \rightarrow x$ then $x_{n} \leq x$ for all $n \in N$;

(2) If $\left\{y_{n}\right\}$ is an decreasing sequence with $y_{n} \rightarrow y$ then $y \leq y_{n}$ for all $n \in N$.

Then $f$ and $g$ have a coupled common fixed point in $X$.

Proof. As in Theorem 4, we can construct two sequences $\left\{x_{n}\right\}$ and $\left\{y_{n}\right\}$ and using (2.10), we have

$$
\begin{aligned}
& \int_{0}^{S\left(f\left(x_{2 n}, y_{2 n}\right), f\left(x_{2 n}, y_{2 n}\right), g\left(x_{2 n+1}, y_{2 n+1}\right)\right)} \varphi(t) d t \\
& \leq \phi \int_{0}^{\left(\frac{s\left(x_{2 n}, x_{2 n}, x_{2 n+1}\right)+S\left(y_{2 n}, y_{2 n}, y_{2 n+1}\right)}{2}\right)} \varphi(t) d t \\
& \int_{0}^{S\left(x_{2 n+1}, x_{2 n+1}, x_{2 n+2}\right)} \varphi(t) d t<\int_{0}^{\left(\frac{S\left(x_{2 n}, x_{2 n}, x_{2 n+1}\right)+S\left(y_{2 n}, y_{2 n}, y_{2 n+1}\right)}{2}\right)} \varphi(t) d t,
\end{aligned}
$$


This gives,

$$
S\left(x_{2 n+1}, x_{2 n+1}, x_{2 n+2}\right)<\left(\frac{S\left(x_{2 n}, x_{2 n}, x_{2 n+1}\right)+S\left(y_{2 n}, y_{2 n}, y_{2 n+1}\right)}{2}\right) .
$$

In the same way, we get

$$
S\left(y_{2 n+1}, y_{2 n+1}, y_{2 n+2}\right)<\left(\frac{S\left(y_{2 n}, y_{2 n}, y_{2 n+1}\right)+S\left(x_{2 n}, x_{2 n}, x_{2 n+1}\right)}{2}\right) .
$$

On adding the above inequalities and using the properties of function $\phi$, we get $\left\{t_{n}\right\}$ be a decreasing sequence and $\lim _{n \rightarrow \infty} t_{n}=0$.

Again, by using the properties of S-metric space we observe that $\left\{x_{n}\right\}$ and $\left\{y_{n}\right\}$ are the Cauchy sequences in X. By continuity of function $\mathrm{f}$, we have $x=f(x, y)$ and $y=f(y, x)$.

Now from (2.10), we get

$$
\int_{0}^{S(f(x, y), f(x, y), g(x, y))} \varphi(t) d t \leq \phi \int_{0}^{\left(\frac{S(x, x, x)+S(y, y, y)}{2}\right)} \varphi(t) d t,
$$

which implies $S(x, x, g(x, y))=0$ or $g(x, y)=x$. Similarly $g(y, x)=y$.

Thus $(x, y)$ is the coupled common fixed point of $\mathrm{f}$ and $\mathrm{g}$.

Now, assuming the condition that for increasing sequence $\left\{x_{n}\right\}$ with $x_{n} \rightarrow x$ then $x_{n} \leq x$ and for decreasing sequence $\left\{y_{n}\right\}$ with $y_{n} \rightarrow y$ then $y_{n} \geq y$ for all $n \in N$,

$$
\begin{aligned}
\int_{0}^{S(x, x, f(x, y))} \varphi(t) d t \leq \int_{0}^{2 S\left(x, x, x_{n}\right)} \varphi(t) d t+\int_{0}^{S\left(f(x, y), f(x, y), x_{n}\right)} \varphi(t) d t \\
\quad=\int_{0}^{2 S\left(x, x, x_{n}\right)} \varphi(t) d t+\int_{0}^{S\left(f(x, y), f(x, y), g\left(x_{n-1}, y_{n-1}\right)\right)} \varphi(t) d t \\
\quad \leq \int_{0}^{2 S\left(x, x, x_{n}\right)} \varphi(t) d t+\phi \int_{0}^{\left(\frac{S\left(x, x, x_{n-1}\right)+S\left(y, y, y_{n-1}\right)}{2}\right)} \varphi(t) d t .
\end{aligned}
$$

Taking limit as $n \rightarrow \infty$, we obtain

$$
\int_{0}^{S(x, x, f(x, y))} \varphi(t) d t \leq \int_{0}^{2 S(x, x,)} \varphi(t) d t+\phi \int_{0}^{\left(\frac{S(x, x, x)+S(y, y, y)}{2}\right)} \varphi(t) d t,
$$

This shows that $f$ has coupled fixed point. By interchanging the role of mappings $f$ and $\mathrm{g}$, we get the coupled fixed point of $\mathrm{g}$.

Hence, we conclude that in both cases $\mathrm{f}$ and $\mathrm{g}$ have coupled fixed point. 


\section{REFERENCES}

[1] I. Altun, "Common fixed point theorems for weakly increasing mappings on ordered uniform spaces," Miskolc Math. Notes, vol. 12, no. 1, pp. 3-10, 2011.

[2] H. Aydi, "On common fixed point theorems for generalized weakly contractive mappings," Miskolc Math. Notes, vol. 14, no. 1, pp. 19-30, 2013.

[3] D. Delbosco, "Un'estensione di un teorema sul punto di S. Reich," Rend. Sem. Mat. Univers. Politecn. Torino, vol. 35, pp. 233-238, 1967.

[4] B. C. Dhage, "Generalized metric spaces and mapping with fixed point," Bull. Calcutta Math. Soc., vol. 84, pp. 329-336, 1992.

[5] B. Fisher, "Mappings with a common fixed point," Math. Sem. Notes Kobe Univ., vol. 7, pp. 115$148,1979$.

[6] S. Gahler, "2-metricsche raume und ihre topologische structure," Math. Nachr., vol. 26, pp. 115$148,1963$.

[7] M. E. Gordji, E. Akbartabar, Y. J. Cho, and M. Remezani, "Coupled common fixed point theorems for mixed weakly monotone mappings in partially ordered metric spaces," Fixed Point Theory and Applications, vol. 95, 2012.

[8] V. Gupta and N. Mani, "Existence and uniqueness of fixed point for contractive mapping of integral type," International Journal of Computing Science and Mathematics, vol. 4, no. 1, pp. 72 - 83, 2013.

[9] V. Gupta, N. Mani, and A. K. Tripathi, "A fixed point theorem satisfying a generalized weak contractive condition of integral type," International Journal of Mathematical Analysis, vol. 6, pp. $1883-1889,2012$.

[10] K. S. Ha, Y. J. Cho, and A. White, "Strictly convex and 2-convex 2-normed spaces," Math. Japonica, vol. 33(3), pp. 375-384, 1988.

[11] K. Iseki, “On common fixed point of mappings," Mathematics Seminar notes, vol. 2(14), 1974.

[12] K. Iseki, "Fixed point theorems in 2-metric spaces," Math. semin. Notes, Kobe Univ., vol. 3, pp. 133-136, 1975.

[13] G. Jungck, "Commuting mappings and fixed points," American Mathematical Monthly, vol. 3, pp. 261-263, 1978.

[14] M. S. Khan, "On the convergence of sequences of fixed points in 2-metric spaces," Indian Journal of Pure and Applied Mathematics, vol. 10(9), pp. 1062-1067, 1979.

[15] M. S. Khan, M. Swalesh, and S. Sessa, "Fixed point theorems by altering distances between the points," Bull. Austral. Math. Soc., vol. 30, pp. 323-326, 1984.

[16] T. Kubiak, "Common fixed points of pairwise commuting mappings," Math. Nachr., vol. 118, pp. 123-127, 1984.

[17] V. Lakshmikantham and L. Ciric, "Coupled common fixed point theorems for nonlinear contractions inpartially orederd metric spaces," Nonlinear Analysis, vol. 70, pp. 4341-4349, 2009.

[18] R. D. Marr, "Partially ordered space and metric spaces," American Mathematical Monthly, vol. 72(6), pp. 628-631, 1965

[19] P. Murthy, S. Chang, Y. J. Cho, and B. K. Sharma, "Compatible mappings of type A and common fixed point theorem," Kyungpook Math., vol. 32, pp. 203-216, 1992.

[20] Z. Mustafa and B. Sims, "Some remarks concerning D-metric spaces," Proceedings of the International Conferences on Fixed Point Theory and Applications, Valencia(Spain), pp. 189-198, 2003.

[21] Z. Mustafa and B. Sims, "A new approach to generalized metric spaces,", J. Nonlinear and Convex Anal., vol. 7(2), pp. 289-297, 2006.

[22] S. Sedghi, N. Shobe, and H. Zhou, "A common fixed point theorem in $D^{*}$ metric spaces," Fixed Point theory and Application, vol. Article ID 27906, p. 13 pages, 2007. 
[23] S. Sedghi and N. Shobe, "A common unique random fixed point theorems in S-metric spaces," Journal of Prime Research in Mathematics, vol. 7, pp. 25-34, 2011.

[24] S. Sedghi, N. Shobe, and A. Aliouche, "A generalizations of fixed point theorems in S-metric spaces," Matematnhpn Bechnk, vol. 64, no. 3, pp. 258-266, 2012.

[25] N. Van Dung, "On coupled common fixed points for mixed weakly monotone maps in partially ordered S-metric spaces," Fixed Point Theory and Applications, vol. 48, p. 17 pages, 2013.

\section{Authors' addresses}

\section{Vishal Gupta}

Department of Mathematics, Maharishi Markandeshwar University, 1317/1, Hargolal Tower Street, Hargolal Road, Ambala Cantt., Haryana, India

E-mail address: vishal.gmn@gmail.com

\section{Raman Deep}

Department of Mathematics, Maharishi Markandeshwar University, Mullana, Ambala, Haryana, India

E-mail address: ramandeepvirk02@gmail.com 